\title{
THE MPIFR RADIO CONTINUUM SURVEYS AND THEIR WWW DISTRIBUTION
}

\author{
E. FÜRST, W. REICH, P. REICH, \\ B. UYANIKER AND R. WIELEBINSKI \\ Max-Planck-Institut für Radioastronomie, Bonn
}

\section{Introduction}

The observation of an area of $120^{\circ} \times 56^{\circ}$ centered on $\mathrm{RA}=8^{h}, \mathrm{DEC}=20^{\circ}$ at $408 \mathrm{MHz}$ was the first astronomical use of the MPIfR 100-m telescope (1970) and was designed to compile a complete sky survey using also data from Jodrell Bank and Parkes (Haslam et al., 1982). The observation of the northern sky at $1420 \mathrm{MHz}$ started in 1972 using the Stockert 25-m telescope and was finished in 1976 (Reich and Reich 1986). This survey has been completed to an all sky survey using data from Villa Elisa (Argentina). The two surveys are absolutely calibrated. The angular resolutions are $0.8^{\circ}$ and $0.59^{\circ}$, respectively. A number of surveys of the Galactic plane have been made with the 100-m telescope at arc minute angular resolution. Surveys at $2695 \mathrm{MHz}\left(|b| \leq 5^{\circ}\right)$ (Reich et al. 1990 , Fürst et al. 1990) and at $1410 \mathrm{MHz}\left(|b| \leq 4^{\circ}\right.$ ) (Reich et al. 1990) are public.

At medium Galactic latitudes (up to $|b|=20^{\circ}$ ) the emission consists mainly of faint extended ridges or arcs superimposed on the still dominating, about 10 times stronger, diffuse Galactic emission. They have never been investigated in a systematic way although they provide important clues for the understanding of the "disk-halo connection". This region is covered by new observations at $1400 \mathrm{MHz}$ with the $100-\mathrm{m}$ telescope.

\section{A New $1400 \mathrm{MHz}$ Survey with the 100-m Telescope}

The new survey covers the accessible Galactic area for the latitude range $4^{\circ} \leq|b| \leq 20^{\circ}$ in total power and linear polarization. The survey will complement the running $21 \mathrm{~cm}$ VLA survey (Condon et al.) for the missing extended predominantly Galactic component. The sensitivity is about 

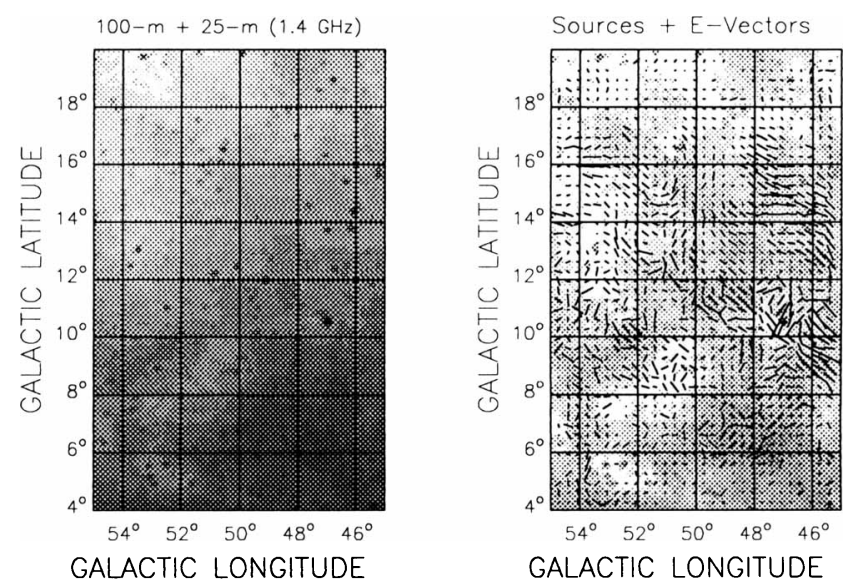

Figure 1. Part of the new $1400 \mathrm{MHz}$ Survey. Left panel: data on an absolute scale. Right panel: The diffuse Galactic emission removed, polarization E-vectors superimposed.

$15 \mathrm{mK}$ r.m.s. in $\mathrm{T}_{B}$, close to the confusion limit. The data will be adjusted to the absolutely calibrated $1420 \mathrm{MHz}$ northern sky survey made with the Stockert 25-m telescope. It is also planned to combine these data with the VLA data to obtain a survey at an angular resolution of about one arc minute. The polarization $\mathrm{U}$ and $\mathrm{Q}$ will be adjusted using the polarization data obtained with the Dwingeloo 25-m telescope (Brouw and Spoelstra 1976), wherever it is possible.

\subsection{FIRST RESULTS}

About $15 \%$ of the survey has been completed. The first example is shown in Figure 1. The left panel shows the new 100-m data. Large-scale structures down to $3^{\circ}$ have been removed in the right panel where polarization vectors are superimposed. A large number of emission structures are visible up to $b=20^{\circ}$. The polarized emission seems to conincide with these structures, but part of it may be associated with the removed diffuse emission. To distinguish between local and distant structures, the comparison with HI surveys will be important. For a distance of $1 \mathrm{kpc}$ the observed emission structures at $b \approx 15^{\circ}$ are about $250 \mathrm{pc}$ above the Galactic plane. A substantial energy is required to push these structures out of the plane.

The second example (Figure 2) demonstrates the combination of the $100-\mathrm{m}$ and the VLA data. The left panel (single dish data) shows the general decline of the Galactic disk emission away from the plane and a faint partial shell of about $200 \mathrm{mK} \mathrm{T} B$ brightness temperature. This emission is not visible in VLA data (middle panel). This data shows a great number of compact sources. The combination of both data sets is shown in the 

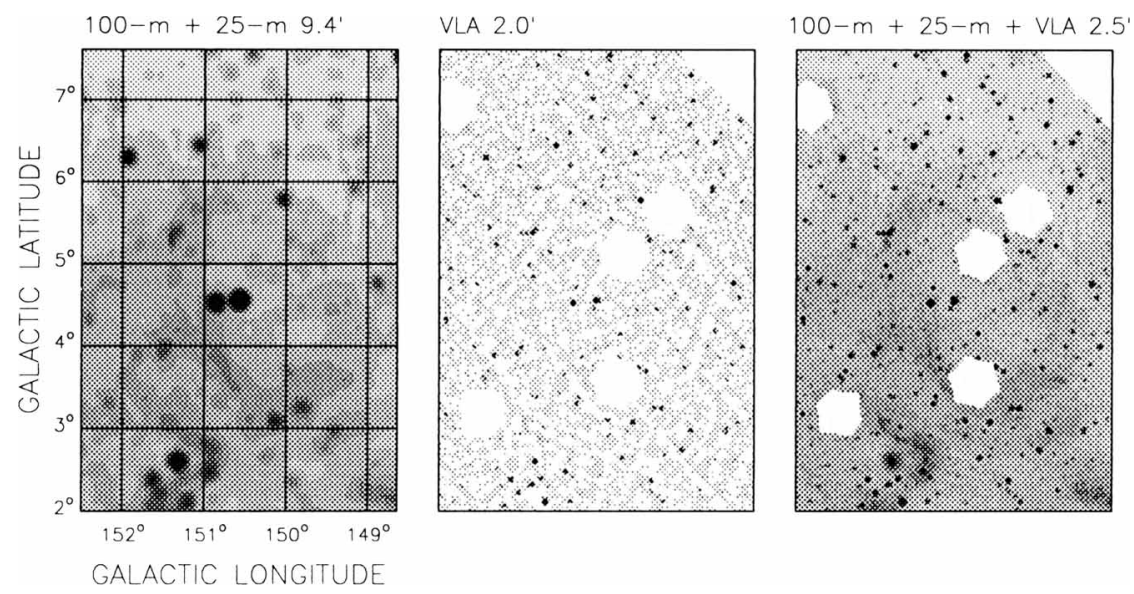

Figure 2. Left panel: $1400 \mathrm{MHz}$ data on an absolute scale. Middle panel: The VLA $1400 \mathrm{MHz}$ data. Right panel: Both data sets combined.

right panel. After subtraction of the compact sources from the combined map flux densities and spectral indices of diffuse emission structures can be accurately determined.

\section{WWW}

All the radio continuum surveys mentioned in this report are or will be available to the community via the WWW. A special retrieval software is offered at http://www.mpifr-bonn.mpg.de/survey.html. It is planned to include pulsar and spectral line data later.

\section{References}

Brouw, W.N., Spoelstra, T.A.Th. (1976) Linear Polarization of the Galactic Background at frequencies between $408 \mathrm{MHz}$ and $1411 \mathrm{MHz}$. Reductions Astron.Astrophys.Suppl. $26,129$.

Fürst, E., Reich, W., Reich, P., Reif, K. (1990) A radio continuum survey of the Galactic Plane at $11 \mathrm{~cm}$ wavelength. II. The area $76^{\circ} \leq l \leq 240^{\circ},-5^{\circ} \leq b \leq 5^{\circ}$ Astron.Astrophys.Suppl. 85,691.

Haslam, C.G.T., Salter, C.J., Stoffel, H., Wilson, W.E. (1982) A 408 MHz All-Sky Continuum Survey. II. The Altas of Contour Maps, Astron.Astrophys.Suppl. 47, 1.

Reich, P., Reich, W. (1986) A Radio Continuum Survey of the northern Sky at $1420 \mathrm{MHz}$ - Part II Astron.Astrophys.Suppl. 63, 205.

Reich, W., Reich, P., Fürst, E. (1990) The Effelsberg $21 \mathrm{~cm}$ radio continuum survey of the Galactic plane between $l=357^{\circ}$ and $l=95.5^{\circ}$ Astron.Astrophys.Suppl. 83, 539 .

Reich, W., Fürst, E., Reich, P., Reif, K. (1990) A radio continuum survey of the Galactic Plane at $11 \mathrm{~cm}$ wavelength. II. The area $358^{\circ} \leq l \leq 76^{\circ},-5^{\circ} \leq b \leq 5^{\circ}$ Astron.Astrophys.Suppl. 85, 633. 